\title{
Erratum to: Peer Victimization and Educational Outcomes in Mainstreamed Adolescents with Autism Spectrum Disorder (ASD)
}

\author{
Ryan Adams $^{1} \cdot$ Julie Taylor $^{2} \cdot$ Amie Duncan $^{1} \cdot$ Somer Bishop $^{3}$
}

Published online: 15 October 2016

(C) Springer Science+Business Media New York 2016

\section{Erratum to: J Autism Dev Disord DOI 10.1007/s10803-016-2893-3}

In the original publication, the asterisks $(* * *)$ were incorrectly added in the body of the Tables 2,3 and 4 . The single, double and triple asterisks were inadvertently replaced with triple asterisks during the production process. The original article was corrected.

The online version of the original article can be found under doi:10.1007/s10803-016-2893-3.

Ryan Adams

ryan.adams@cchmc.org; Ryan.Adams@ccmc.org

1 Division of Developmental and Behavioral Pediatrics, Cincinnati Children's Hospital Medical Center, 3333 Burnet Avenue, MLC 4002, Cincinnati, OH 45229, USA

2 Vanderbilt University, Nashville, TN, USA

3 University of California San Francisco, San Francisco, CA, USA
Table 2 Findings of multiple regressions examining the associations between peer victimization and educational outcomes outcomes for Study 1

\begin{tabular}{|c|c|c|c|c|}
\hline & $\mathrm{b}$ & t-value & $\beta$ & $\Delta R 2$ \\
\hline \multicolumn{5}{|l|}{ Enjoys school } \\
\hline Step 1 & & & & 0.00 \\
\hline Age & 0.01 & 0.66 & 0.03 & \\
\hline Gender & 0.18 & 1.55 & 0.08 & \\
\hline Step 2 & & & & $0.09 * * *$ \\
\hline Verbal victimization & 0.53 & $6.51 * * *$ & 0.30 & \\
\hline Step 2 & & & & $0.10 * * *$ \\
\hline Ignored & 0.61 & $7.07 * * *$ & 0.33 & \\
\hline Step 2 & & & & $0.03 * *$ \\
\hline Physical victimization & 0.37 & $3.43 * *$ & 0.16 & \\
\hline Step 2 & & & & $0.05 * * *$ \\
\hline Provoke & 0.41 & $4.81 * * *$ & 0.23 & \\
\hline \multicolumn{5}{|l|}{ Belong to school } \\
\hline Step 1 & & & & 0.01 \\
\hline Age & 0.03 & 1.51 & 0.07 & \\
\hline Gender & 0.18 & 1.57 & 0.08 & \\
\hline Step 2 & & & & $0.13 * * *$ \\
\hline Verbal victimization & 0.61 & $7.86 * * *$ & 0.36 & \\
\hline Step 2 & & & & $0.12 * * *$ \\
\hline Ignored & 0.62 & $7.30 * * *$ & 0.34 & \\
\hline Step 2 & & & & $0.06 * * *$ \\
\hline Physical victimization & 0.51 & $4.80 * * *$ & 0.23 & \\
\hline Step 2 & & & & $0.05 * * *$ \\
\hline Provoke & 0.39 & $4.64 * * *$ & 0.22 & \\
\hline \multicolumn{5}{|l|}{ Safe at school } \\
\hline Step 1 & & & & 0.00 \\
\hline Age & 0.01 & 0.69 & 0.03 & \\
\hline Gender & 0.05 & 0.48 & 0.02 & \\
\hline
\end{tabular}


Table 2 continued

\begin{tabular}{|c|c|c|c|c|}
\hline & $\mathrm{b}$ & t-value & $\beta$ & $\Delta R 2$ \\
\hline Step 2 & & & & $0.21 * * *$ \\
\hline Verbal victimization & 0.67 & $10.76^{* * *}$ & 0.47 & \\
\hline Step 2 & & & & $0.14 * * *$ \\
\hline Ignored & 0.61 & $8.59 * * *$ & 0.39 & \\
\hline Step 2 & & & & $0.14 * * *$ \\
\hline Physical victimization & 0.73 & $8.55^{* * *}$ & 0.39 & \\
\hline Step 2 & & & & $0.10 * * *$ \\
\hline Provoke & 0.49 & $7.06 * * *$ & 0.33 & \\
\hline \multicolumn{5}{|l|}{ Academic performance } \\
\hline Step 1 & & & & 0.00 \\
\hline Age & 0.03 & 0.80 & 0.04 & \\
\hline Gender & 0.12 & 0.70 & 0.03 & \\
\hline Step 2 & & & & 0.00 \\
\hline Verbal victimization & 0.11 & 0.87 & 0.04 & \\
\hline Step 2 & & & & 0.00 \\
\hline Ignored & 0.04 & 0.32 & 0.02 & \\
\hline Step 2 & & & & 0.00 \\
\hline Physical victimization & 0.13 & 0.79 & 0.04 & \\
\hline Step 2 & & & & 0.00 \\
\hline Provoke & 0.09 & 0.69 & 0.03 & \\
\hline
\end{tabular}

$* \mathrm{p}<0.05, * * \mathrm{p}<0.01, * * * \mathrm{p}<0.001$
Table 3 Findings of multiple regressions examining the associations between peer victimization and educational outcomes

\begin{tabular}{|c|c|c|}
\hline & Log ratio & Wald \\
\hline \multicolumn{3}{|l|}{ Academic problems } \\
\hline \multicolumn{3}{|l|}{ Step 1} \\
\hline Age & 1.21 & $12.75^{* * *}$ \\
\hline Gender & 0.81 & 0.56 \\
\hline \multicolumn{3}{|l|}{ Step 2} \\
\hline Verbal victimization & 1.39 & $2.76^{*}$ \\
\hline \multicolumn{3}{|l|}{ Step 2} \\
\hline Ignored & 1.64 & $5.21^{* *}$ \\
\hline \multicolumn{3}{|l|}{ Step 2} \\
\hline Physical victimization & 0.95 & 0.02 \\
\hline \multicolumn{3}{|l|}{ Step 2} \\
\hline Provoke & 1.38 & $2.47 *$ \\
\hline \multicolumn{3}{|l|}{ Social problems } \\
\hline \multicolumn{3}{|l|}{ Step 1} \\
\hline Age & 1.03 & 0.21 \\
\hline Gender & 0.60 & $2.85^{*}$ \\
\hline \multicolumn{3}{|l|}{ Step 2} \\
\hline Verbal victimization & 2.18 & $10.77^{* *}$ \\
\hline \multicolumn{3}{|l|}{ Step 2} \\
\hline Ignored & 2.66 & $12.39^{* * *}$ \\
\hline \multicolumn{3}{|l|}{ Step 2} \\
\hline Physical victimization & 1.19 & 0.21 \\
\hline \multicolumn{3}{|l|}{ Step 2} \\
\hline Provoke & 4.07 & $35.90 * * *$ \\
\hline
\end{tabular}


Table 4 Findings of multiple regressions examining the associations between peer victimization and educational outcomes for Study 2

b $\quad t$-value $\quad \beta \quad \Delta R 2$

Disobedient at school

\begin{tabular}{|c|c|c|c|c|}
\hline Step 1 & & & & 0.02 \\
\hline Age & -0.03 & -0.76 & -0.11 & \\
\hline Income & -0.02 & -0.33 & -0.07 & \\
\hline Step 2 & & & & $0.33 * * *$ \\
\hline Verbal victimization & 0.18 & $4.93 * * *$ & 0.59 & \\
\hline Step 2 & & & & $0.15 * *$ \\
\hline Relational victimization & 0.12 & $2.97 * *$ & 0.41 & \\
\hline Step 2 & & & & 0.04 \\
\hline Physical victimization & 0.10 & 1.38 & 0.21 & \\
\hline Step 2 & & & & $0.40 * * *$ \\
\hline ASD victimization & 0.20 & $5.81 * * *$ & 0.67 & \\
\hline \multicolumn{5}{|l|}{ Fears going to school } \\
\hline Step 1 & & & & 0.02 \\
\hline Age & 0.03 & 0.95 & 0.12 & \\
\hline Income & -0.02 & -0.42 & -0.08 & \\
\hline Step 2 & & & & $0.08^{*}$ \\
\hline Verbal victimization & 0.07 & $2.11 *$ & 0.30 & \\
\hline Step 2 & & & & 0.04 \\
\hline Relational victimization & 0.03 & 1.34 & 0.20 & \\
\hline Step 2 & & & & 0.02 \\
\hline Physical victimization & 0.06 & 0.98 & 0.14 & \\
\hline Step 2 & & & & 0.05 \\
\hline ASD victimization & 0.05 & 1.62 & 0.23 & \\
\hline \multicolumn{5}{|l|}{ Poor school work } \\
\hline Step 1 & & & & 0.02 \\
\hline Age & -0.03 & -0.73 & -0.10 & \\
\hline Income & -0.04 & -1.71 & -0.13 & \\
\hline Step 2 & & & & $0.12 *$ \\
\hline Verbal victimization & 0.14 & $2.53^{*}$ & 0.35 & \\
\hline Step 2 & & & & 0.04 \\
\hline Relational victimization & 0.07 & 1.38 & 0.21 & \\
\hline Step 2 & & & & 0.00 \\
\hline Physical victimization & 0.02 & 0.16 & 0.03 & \\
\hline Step 2 & & & & $0.13 * *$ \\
\hline ASD victimization & 0.15 & $2.82 * *$ & 0.39 & \\
\hline
\end{tabular}

${ }^{*} \mathrm{p}<0.05,{ }^{* *} \mathrm{p}<0.01,{ }^{* * *} \mathrm{p}<0.001$ 